TITLE:

\title{
Observation of cell shapes in wood cross-sections during water adsorption by confocal laser- scanning microscopy (CLSM)
}

\section{AUTHOR(S):}

Taguchi, Ayako; Murata, Koji; Nakano, Takato

\section{CITATION:}

Taguchi, Ayako ... [et al]. Observation of cell shapes in wood cross-sections during water adsorption by confocal laser-scanning microscopy (CLSM). Holzforschung 2010, 64(5): 627-631

ISSUE DATE:

2010-08

URL:

http://hdl.handle.net/2433/141947

\section{RIGHT:}

C 2007-2011. Walter de Gruyter GmbH \& Co. KG.; 許諾条件により本文は 2011-09-01に公開 


\section{Observation of cell shapes in wood cross-sections during water adsorption by confocal laser-scanning microscopy (CLSM)}

\author{
Ayako Taguchi, Koji Murata and Takato Nakano* \\ Laboratory of Biomaterials Design, Division of Forest and \\ Biomaterials Science, Graduate School of Agriculture, \\ Kyoto University, Kyoto, Japan \\ *Corresponding author \\ Laboratory of Biomaterials Design, Division of Forest and \\ Biomaterials Science, Graduate School of Agriculture, Kyoto \\ University, Kita-Shirakawa, Kyoto, 606-8502, Japan \\ E-mail: tnakano@kais.kyoto-u.ac.jp
}

\begin{abstract}
Deformation behaviors of cell cross-sections from wood blocks were observed during water adsorption by confocal laser-scanning microscopy. In the tangential (T) direction, cells swelled linearly with increasing moisture content, whereas in the radial $(\mathrm{R})$ direction, cell deformation varied from cell to cell, and some cells showed even shrinkage. This confirms that the well-known swelling/shrinking anisotropy for intact wood blocks is $\mathrm{T}>\mathrm{R}$.
\end{abstract}

Keywords: anisotropic swelling; cell shape; confocal laserscanning microscopy (CLSM); water adsorption.

\section{Introduction}

One of the characteristics of wood is its anisotropic swelling and shrinkage behavior during adsorption or desorption of water below the fiber saturation point (FSP). The wellknown anisotropy ratio in whole wood (as a bulk), in general, is: $10(\mathrm{~T})$ : $5(\mathrm{R})$ : 0.5-1.0 (L). Many mechanisms have been proposed to explain this phenomenon: (1) interaction between earlywood (EW) and latewood (LW; Pentoney 1953), (2) restriction by ray tissues (McIntosh 1957), and (3) differences in microfibril angle in the S2 layer (Barber and Meylan 1964). The examination of the swelling behavior of wood at the level of individual cells, namely, cell cross-sections, could perhaps contribute to the understanding of transverse swelling and shrinking anisotropy. Nakato (1958) observed shrinkage of cells with light microscopy and thin cross-sections, but the methodology does not sufficiently reflect cellular tissue structure related to the swelling of the whole wood as a bulk. Watanabe et al. (1998) investigated shrinkage behavior based on the replica method, in which the surface of wood blocks is observed and cell shapes are compared under oven-dried conditions and in a fully swollen state (if the moisture content is above the FSP).

The swelling behavior can be better understood if the dimensional changes in a whole wood block are observed as a function of moisture content (MC). Confocal laser-scanning microscopy (CLSM) has been well studied for this purpose. Sakagami et al. (2007) used CLSM to visualize the shrinkage behavior of tracheid cells during desorption over time. However, the range of MC investigated by these researchers was an initial MC of $25-30 \%$ and a final MC of $8-10 \%$, which was beyond the critical MC 5\%. The critical point of water adsorption of wood is MC 5\% and many physical properties of wood change at this point (Kollmann and Krech 1960; James 1961). Thus, it is important to observe dimensional change in a $\mathrm{MC}$ range below and beyond $5 \%$. This is the reason why this requirement was fulfilled in the present study.

\section{Materials and methods}

\section{Specimen preparation}

Wood blocks (yezomatsu, Picea jezoensis Carr) of $20 \mathrm{~mm}$ $(t) \times 20 \mathrm{~mm}(r) \times 5 \mathrm{~mm}(l)$ were cut. A transverse surface was cut across each block to obtain a surface of approximately $10 \times 10 \mathrm{~mm}^{2}$. After softening by soaking in distilled water under vacuum, the transverse surfaces were smoothed with a sliding microtome, and the specimens were dried slowly at room temperature to avoid collapse. For slow drying, the specimens were placed in a desiccator conditioned at $80 \%$ relative humidity (RH) for several days, and then were conditioned further in desiccators conditioned at 71, 57, 33 , and $11 \% \mathrm{RH}$, in order. The specimens were conditioned in a desiccator at $11 \% \mathrm{RH}$ for over 3 weeks, until the MC finally achieved equilibrium. The initial MC before the experiments was approximately $4 \%$.

A pair of these specimens was selected from the four specimens obtained from one block, on the basis of weight. One was used for MC measurement, and the others for CLSM.

\section{CLSM}

Instrument: CLSM, Lasertech (Yokohama, Japan), 1LM21H; the objective lens was placed in a self-made environmental chamber made of a metal box with a stage for the specimen, in which a vessel was also situated (containing a saturated solution) under the stage. The specimen was first placed in the chamber with a saturated solution vessel giving a $\mathrm{RH}$ of $11 \%$, and an initial image was taken. Then, the solution was replaced with a solution giving a chamber RH of $47 \%$, and images were taken every $5 \mathrm{~min}$. After $1 \mathrm{~h}$, the solution was again replaced to give at RH of $97 \%$, and images were taken at gradually extended intervals. The temperature was constant at $23^{\circ} \mathrm{C}$. During this time, the $\mathrm{MC}$ of the specimen increased gradually. A total of 45 cells were observed. A few specimens were observed to confirm reproducibility. The weight of similar specimens was measured with time under the same humidity conditions. 


\section{Image processing and analysis}

Images from CLSM were processed and analyzed, and cell outlines determined by means of Adobe Photoshop CS1 (Adobe Systems) and Windows XP (Microsoft). Binary images were then obtained by binary and open processing using Pop Imaging (Digital Being Kids) software (Figure 1). On the basis of the binary images, data were calculated concerning the areas of tracheid cell walls and lumens as well as diameters of cells and lumens in the tangential (T) and radial (R) directions.

\section{Results and discussion}

\section{Changes in MC}

For moderate changes in MC, the specimens were subjected to conditioning at $47 \%$ and $97 \% \mathrm{RH}$, giving equilibrium MCs of $8 \%$ and $27 \%$, respectively. Figure 2 shows the change of MC of a specimen with time over the two stages. The rates of change in MC measured for $30 \mathrm{~min}$ after replacement with saturated solutions were $0.06-0.08 \% \mathrm{~min}^{-1}$ (for RH 47\%), i.e., the change in MC was moderate.

\section{Microscopic observations}

An example of the series of recorded images is presented in Figure 3, showing changes in cell shape with increasing MC. However, this change is not visible to the naked eye. Cell wall thickness of EW near an annual ring was too thin to analyze, and in this report, only EW near LW and LW itself were observed. In all cells, $\mathrm{T}$ diameters of the tracheid and lumen increased linearly with increasing MC. By contrast, the area and $\mathrm{R}$ diameter varied from cell to cell. In spite of the increase in MC, a few cells still shrank. The swelling/ shrinkage behavior of many cells changed at approximately MC $8 \%$, e.g., a certain cell swelled again after shrinkage below MC $8 \%$.

1

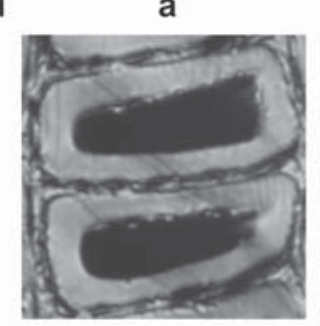

2

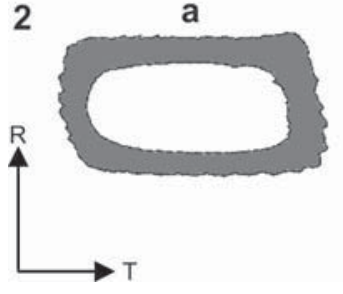

b

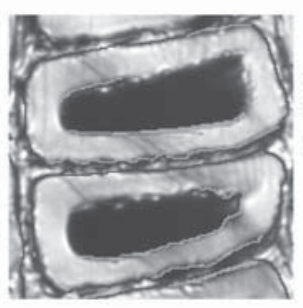

b

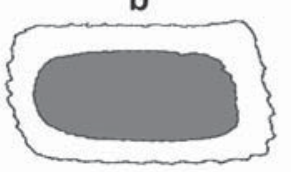

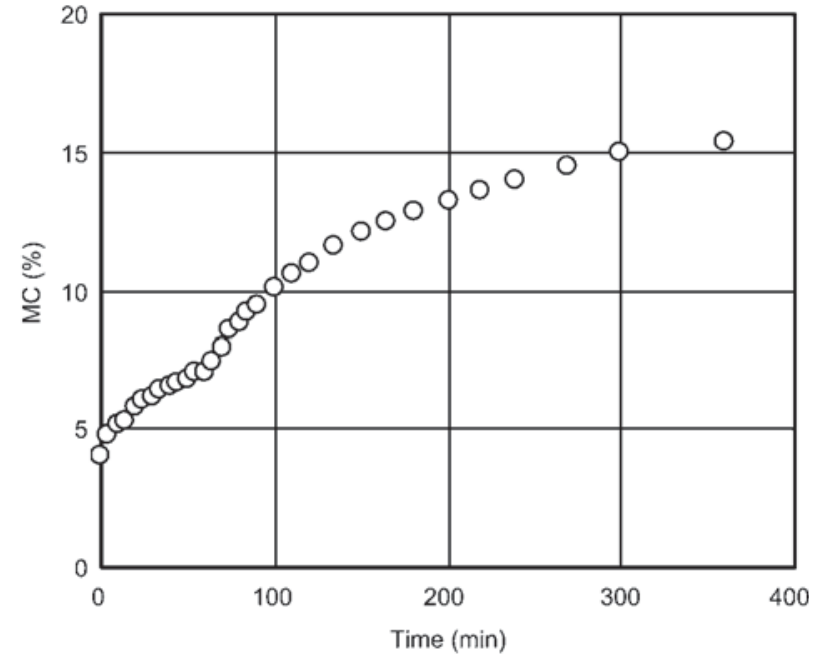

Figure 2 Changes in the MC of a specimen with time.

These changes were confirmed by detailed observation at low MC. In the range of $8-15 \% \mathrm{MC}$, despite differences in shrinkage and swelling, all measurement items (except the T diameter of the lumen) showed a similar tendency to that observed by Sakagami et al. (2007).

\section{Dependence of MC changes in cell area and diameter}

LW In Figure 4, LW shows the typical relations between $\mathrm{MC}$ and dimensional changes in area of tracheid and lumen, taken from the four cells shown in Figure 3a,b. The results are presented as change ratio data which are calculated as ratios on the basis of the initial condition. Deformations of cells in other observed areas also showed the same tendency. The deformation modes of the tracheid areas can be characterized below a MC change of $8 \%$ as swelling, no change, or shrinkage. The tracheid area of all cells increased above
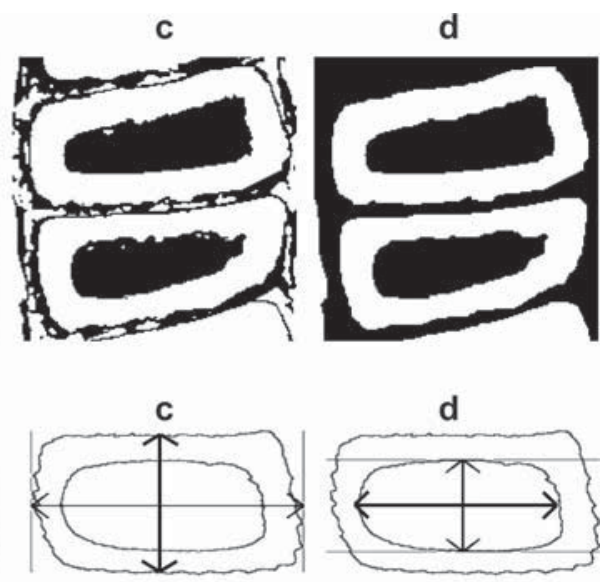

Figure 1 Image processing and analysis procedure. (1) Image process stage: (a) original recorded image; (b) decision on the outline; (c) image after binary process; (d) image after all processes. (2) Data derived from image analysis: (a) tracheid area; (b) lumen area; (c) tracheid diameter in $\mathrm{T}$ and in $\mathrm{R}$ directions; (d) lumen diameter in $\mathrm{T}$ and in $\mathrm{R}$ directions. 


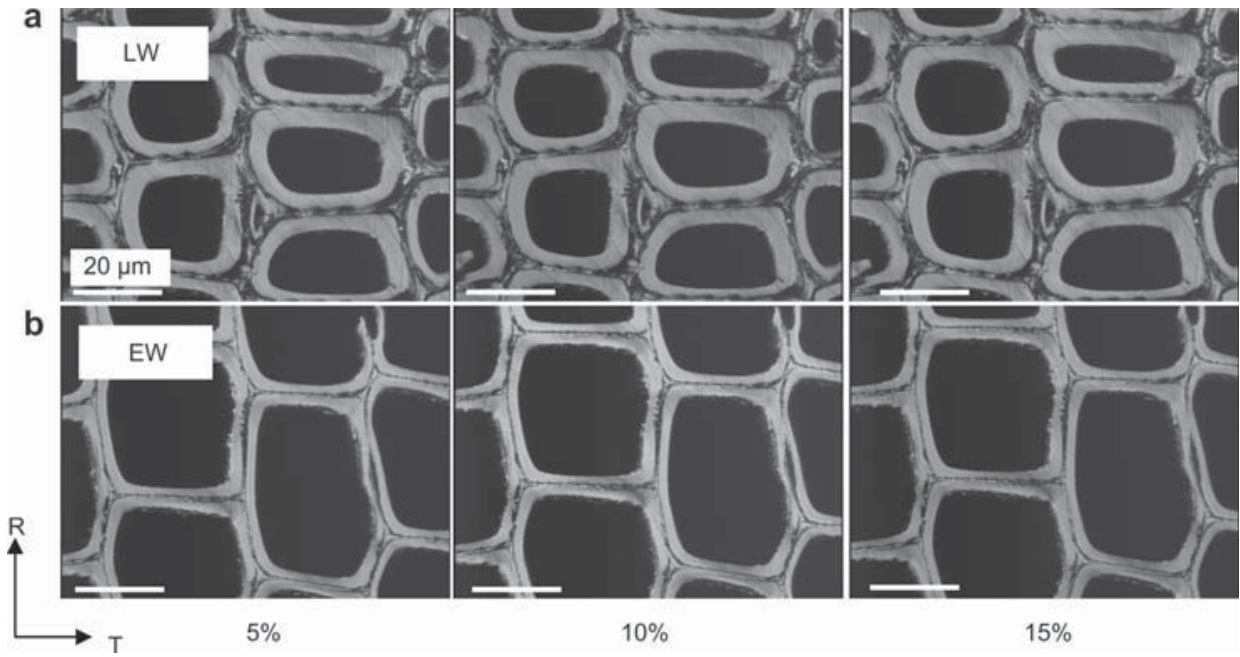

Figure 3 Changes in cell shape with increasing MC in LW and EW.
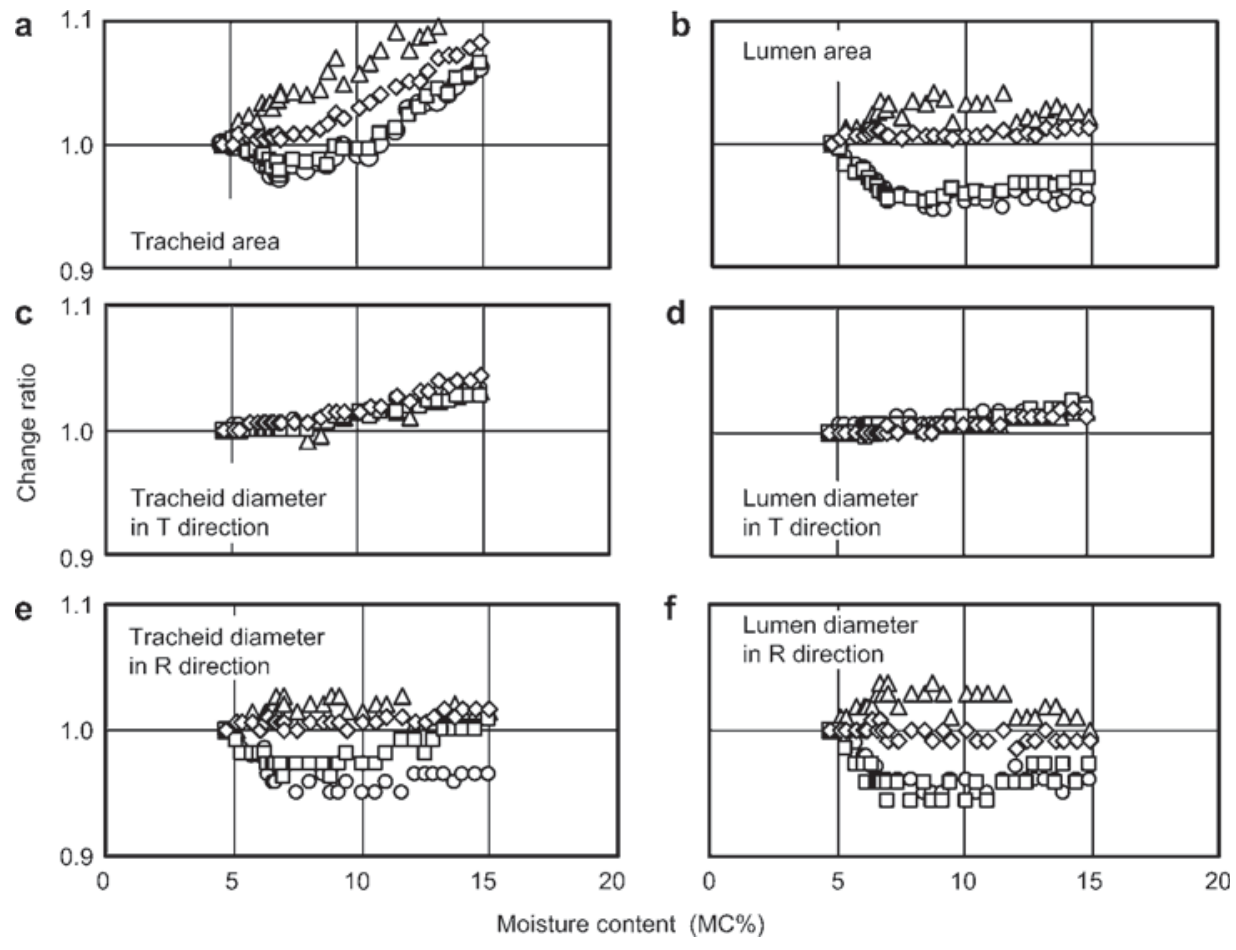

Figure 4 Changes in area and diameter of the tracheid and lumen with increasing MC in LW.

MC $8 \%$ and swelled in comparison with the first and final MCs (Figure 4a). The lumen area below MC $8 \%$ also fell into these three modes; however, above MC $8 \%$ not all cells swelled (Figure 4b). In some cells, both tracheid cell and lumen areas shrank, whereas in other cells they swelled (Figure 4a,b).

Changes in $\mathrm{T}$ and $\mathrm{R}$ diameters of the tracheids and lumens are presented in Figure $4 \mathrm{c}-\mathrm{f}$. The $\mathrm{T}$ diameter increased linearly with increasing MC in all cells. By contrast, there were a few deformation modes for changes in the $\mathrm{R}$ diameter. Below MC $8 \%$, the $\mathrm{R}$ diameter changed as per the lumen area (swelling, no change, or shrinkage); above MC $8 \%$ some cells swelled, whereas others hardly changed. The behaviors of the $\mathrm{R}$ diameter of the tracheid and lumen were similar and affected the area of the lumen. The $\mathrm{R}$ diameter of the tracheids and lumens swelled and shrank more or less intensely. The results are in agreement with those of Watanabe et al. (1998), who compared the R lumen diameter in the oven-dried state and above the FSP.

EW Typical results of EW are given in Figure 5, which were derived from cells shown in Figure 3. In EW, all cells show similar behaviors, unlike in LW. Tracheid and lumen areas decreased to approximately MC $8 \%$, then increased 
a
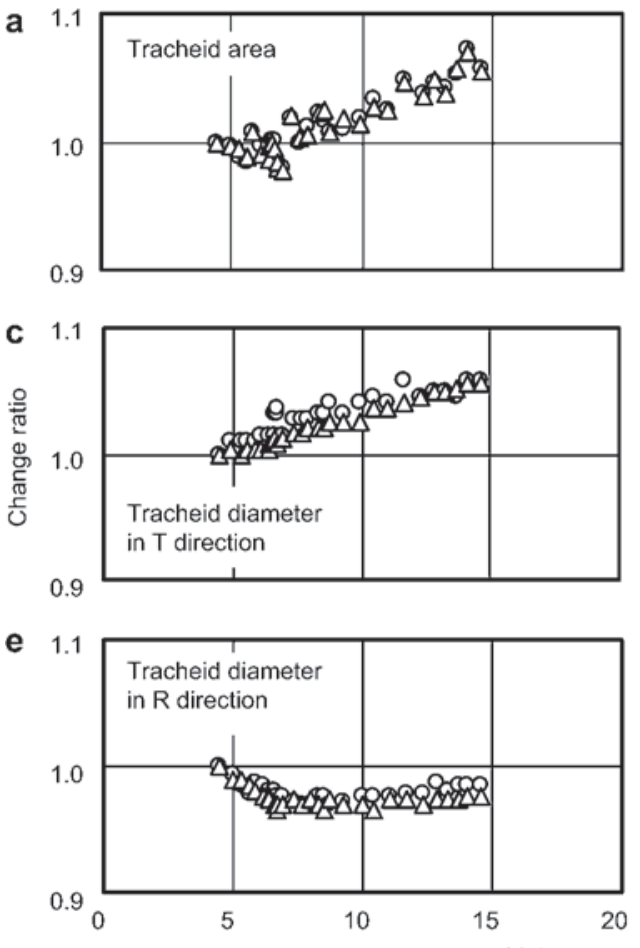
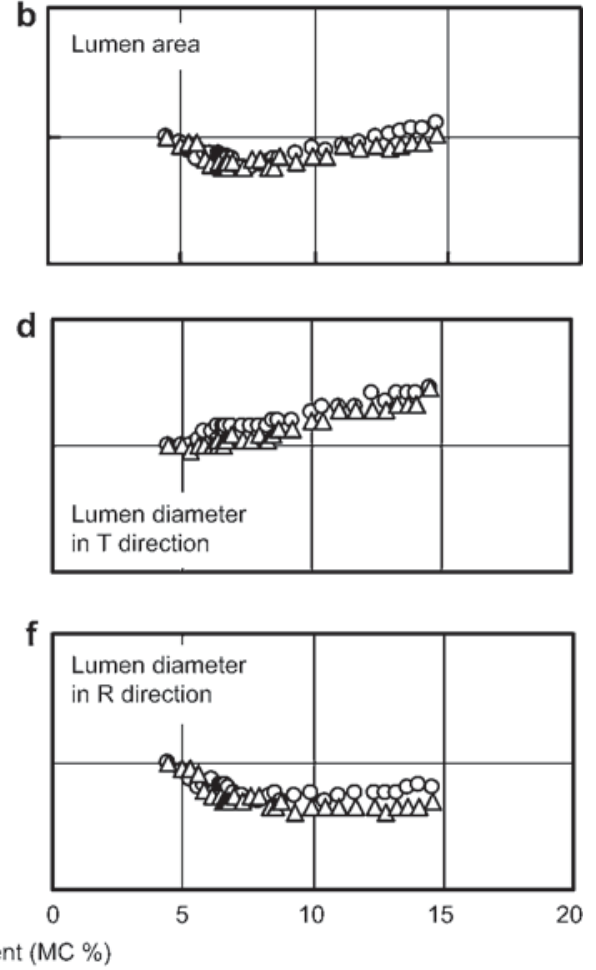

Figure 5 Changes in area and diameter of the tracheid and lumen with increasing MC in EW.

(Figure 5a,b). Tracheid areas almost increased between the first and final MC, but the area of the lumen varied only slightly. Diameters of both the tracheid cell walls and lumens showed the same tendency in the $\mathrm{R}$ and $\mathrm{T}$ directions (Figure $5 \mathrm{c}-\mathrm{f}$ ). In the $\mathrm{T}$ direction, the tracheid and lumen diameters swelled linearly with increasing MC. In the $\mathrm{R}$ direction, diameters of the tracheid and lumen shrank to MC 5-8\%, and above this point maintained the shrinkage state.

Deformations in the tracheid and lumen of EW and LW with increasing MC are characteristic. Chafe and Ilic (1992) noted that the area of the lumen decreased from FSP to MC by $5 \%$ and increased from MC 5 to $0 \%$, i.e., the lumen area was at a minimum at MC 5\%, because of the nonlinearity of wood shrinkage rate in the low MC range. As shown in Figures $4 \mathrm{a}, \mathrm{b}$ and $5 \mathrm{a}, \mathrm{b}$, in both LW and EW, the lumen areas were at a minimum at MC $8 \%$, which is slightly different from the calculated critical point (MC 5\%) of Chafe and Ilic (1992). Regardless of this small difference, the results of the present study for EW support those of the quoted authors.

Both lumen and tracheid areas showed initial shrinkage in some LW cells. In addition, tracheid and lumen diameters also had initial shrinkage, however, only in the $\mathrm{R}$ not in the $\mathrm{T}$ direction. This is probably owing to the nonlinearity effects in the $\mathrm{R}$ direction as reported by Chafe and Ilic (1992). The random arrangement of the cells in the $\mathrm{T}$ of yezomatsu might also explain the observation of the irregular swelling/shrinking effects in the $\mathrm{R}$ direction in the present study as demonstrated in Figures 4 and 5. The rate of this interaction in
LW is larger because the swelling pressure is larger and the cell wall is thicker in LW.

\section{Conclusions}

Microscopic observations of wood block samples during adsorption, as described above, provide information regarding anisotropic swelling. As shown in Figures $4 \mathrm{c}-\mathrm{f}$ and $5 \mathrm{c}-\mathrm{f}$, diameters of the tracheid and lumen in the $\mathrm{T}$ direction of all cells swelled linearly. However, diameters of the tracheid in the R and lumen of LW deformed irregularly: they swelled, did not change, or shrank. Diameters of the tracheid in the $\mathrm{R}$ of EW shrank in most cases. Altogether, in the R direction the shrinkage was predominant. In summary, at a cellular level, the rate of swelling is $\mathrm{T}>\mathrm{R}$ and confirms the findings of the swelling anisotropy in whole wood. Probably, swelling in the $\mathrm{R}$ direction was constricted because of a Poisson effect of swelling in the $\mathrm{T}$ direction.

\section{References}

Barber, N.F., Meylan, B.A. (1964) The anisotropic shrinkage of wood: a theoretical model. Holzforschung 18:146-156.

Chafe, S.C., Ilic, J. (1992) Shrinkage and collapse in thin sections and blocks of Tasmanian mountain ash regrowth. Part 2: The R-ratio and changes in cell lumen volume. Wood Sci. Technol. 26:181-187. 
James, W.L. (1961) Internal friction and speed in Douglas fir. Forest Prod. J. 11:383-388.

Kollmann, F., Krech, H. (1960) Dynamic measurement of damping capacity and elastic properties of wood. Holz Roh Werkst. 18: 4-54.

McIntosh, D.C. (1957) Transverse shrinkage of red oak and beech. Forest Prod. J. 7:114-120.

Nakato, K. (1958) On the cause of the anisotropic shrinkage and swelling of wood. IX. On the relationship between the microscopic structure and the anisotropic shrinkage in the transverse section (2). Mokuzai Gakkaishi 4:134-141.
Pentoney, R.E. (1953) Mechanisms affecting tangential vs. radial shrinkage. J. For. Prod. Res. Soc. 3:27-32.

Sakagami, H., Matsumura, J., Oda, K. (2007) Shrinkage of tracheid cells with desorption visualized by confocal laser scanning microscopy. IAWA J. 28:29-37.

Watanabe, U., Fujita, M., Norimoto, M. (1998) Transverse shrinkage of coniferous wood cells examined using replica method and power spectrum analyses. Holzforschung 52:200-206.

Received January 28, 2010. Accepted April 23, 2010.

Previously published online July 29, 2010. 